\title{
A Very Rare Cause of Erythema Nodosum: Rotavirus
}

\section{Rotavirüs Kaynaklı Eritema Nodozum}

\author{
Hande Yetişgin', Gülsüm İclal Bayhan² \\ ${ }^{1}$ Clinic of Pediatrics, Yıldırım Beyazit University Yenimahalle Training and Research Hospital, Ankara, Turkey \\ ${ }^{2}$ Clinic of Pediatric Infectious Diseases, Yıldırım Beyazit University Yenimahalle Training and Research Hospital, Ankara, Turkey
}

Cite this article as: Yetişgin H, Bayhan Gi. A very rare cause of erythema nodosum: Rotavirus. J Pediatr Inf 2019;13(3):e129-e131.

\begin{abstract}
Erythema nodosum is the most common form of panniculitis, characterized by subcutaneous, erythematous, painful, tender nodules. Erythema nodosum is a Type IV delayed hypersensitivity reaction caused by infectious etiology (39.5\%) and non-infectious (29.6\%) and idiopathic etiology (25\%). Most common infectious etiology include upper respiratory tract infections, of which streptococcal infections are the most common infections followed by Salmonella, Giardia lamblia, and Yersinia enterocolitica. To our knowledge, erythema nodosum associated with rotavirus has been reported in only one case in the literature. In this report, we present a 3-year-old girl who developed erythema nodosum during the course of rotavirus diarrhea.
\end{abstract}

Keywords: Rotavirus, erythema nodosum, diarrhea

\section{Introduction}

Rotavirus is the leading cause of severe acute gastroenteritis in young children, characterized by nausea, vomiting, and fever lasting for 4-7 days. Although rotavirus mostly manifests with diarrhea, some rotavirus strains have been shown to escape the gastrointestinal tract, thereby causing viremia. Literature indicates that there are a number of non-gastrointestinal manifestations of rotavirus, including febrile/afebrile convulsion, encephalitis, myositis, transient acute flaccid paralysis, myocarditis, pancreatitis, hemophagocytic lympho- histiocytosis, kidney stones, and secondary bacteremia (1-3). To our knowledge, erythema nodosum (EN) associated with rotavirus has been reported in only one case in the literature (4). In this report, we present a 3-year-old girl who developed EN during the course of rotavirus diarrhea.

\section{Case Report}

A previously healthy 3-year-old girl presented to outpatient clinic with a 15-day history of diarrhea which started after vomiting. The patient was reported to have watery diarrhea with no mucus. A stool analysis performed at an outside 
health center indicated 5-6 leukocyte per microscopic field and thus the patient was initiated on a probiotic therapy. On the third day of the therapy, the patient was re-admitted to the outside health center with a temperature of $39^{\circ} \mathrm{C}$ and a repeat stool analysis was performed. The stool microscopy was normal, stool culture was negative and the probiotic therapy was continued with the addition of zinc supplementation. Fever lasted only one day and never repeated. On the $15^{\text {th }}$ day, the patient presented to our outpatient clinic with diarrhea lasting for 15 days, skin rash for three days, and fever for one day. Physical examination revealed multiple EN lesions on bilateral pretibial areas and one lesion on the right wrist. Laboratory parameters were as follows: white blood cell count $12.700 / \mathrm{mm}^{3}$, hemoglobin $10.8 \mathrm{mg} / \mathrm{dL}$, platelet count $320.000 / \mathrm{mm}^{3}$, C-reactive protein (CRP) level $1.26 \mathrm{mg} /$ $\mathrm{dL}$ (0-0.5), erythrocyte sedimentation rate $38 \mathrm{~mm} / \mathrm{h}(0-20)$, Antistreptolysin O Titer (ASO) $51.7 \mathrm{IU} / \mathrm{mL}$ (0-200). Biochemical parameters were normal. Stool microscopy revealed no erythrocyte, leukocyte, parasitic ova, or cyst. Rotavirus antigen was positive. No bacterial growth was detected in the stool culture and throat cultures. The patient was initiated on a nonsteroidal anti-inflammatory therapy. The lesions resolved rapidly and no recurrence was observed during the follow-up period. The patient came to our clinic regularly for one and a half years for follow-up, and diarrhea was never repeated.

\section{Discussion}

Almost all children up to 5 years of age are infected with rotavirus. Typical rotavirus infection is associated with fever, vomiting and diarrhea. Rotavirus infection leads to clinical manifestations in a wide spectrum. During the course of rotavirus infection, subclinical infection, limited duration of watery diarrhea and vomiting, or severe diarrhea and vomiting which can cause electrolyte imbalance, severe dehydration and death could be seen. The duration of illness is commonly 3 to 7 days but in more severe cases can be as long as 2 to 3 weeks. In our cases the patient presented with typical triad for rotavirus infection. Other probable etiological agents were excluded $(5,6)$.

Erythema nodosum is the most common form of panniculitis. Erythema nodosum commonly manifests as erythematous, warm, nonulcerative, and painful lesions on the extensor aspects of lower extremities. These lesions do not cause necrosis or scar formation but may lead to mild hyperpigmentation (7). Erythema nodosum is a Type IV delayed hypersensitivity reaction caused by various etiological factors. EN is mostly caused by infectious etiology (39.5\%) followed by noninfectious (29.6\%) and idiopathic etiology (25\%). The most common infectious cause is upper respiratory tract infections, the most common of which are streptococcal infections. Gastrointestinal tract infections have been reported in $3.7 \%$ of the EN patients, mostly caused by Blastocystis hominis, Helicobacter pylori, Salmonella, Giardia lamblia, and Yersinia enterocolitica (8). Another study showed that the most common factor was streptococcal infections followed by tuberculosis, tularemia, cytomegalovirus infection, sarcoidosis, and Behçet's disease. The study also noted that one patient had EN associated with gastrointestinal infection caused by Giardia lamblia (9).

Skin diseases associated with rotavirus include maculopapular rash, Gianotti Crosti syndrome, and acute infantile hemorrhagic edema $(10,11)$. To our knowledge, there has been only one case reported in the literature who developed EN associated with rotavirus diarrhea. In that case, the EN lesions developed during the course of a mixed infection of rotavirus and salmonella (4).

Mean age of incidence in EN is reported to be 11 years (9). The lesions in EN mostly occur in the pretibial area, with $9.9 \%$ of them reported on the arm, elbow, knee, and femoral region and $17.3 \%$ of them reported in both the pretibial area and other regions (12). Meaningfully, although EN has been mostly reported to occur in school-age children, our patient was a 3-year-old girl. In our patient, the lesions were present both in bilateral pretibial areas and on the right wrist.

In conclusion, the EN lesions in our patient were considered to result from rotavirus since the clinical features of the patient was consistent with viral gastroenteritis, no bacterial growth was detected in the stool and throat cultures, and rotavirus antigen was positive in the stool sample. The case presented in this report implicates that although rotavirus has been shown to cause numerous non-gastrointestinal manifestations, it is likely to cause erythema nodosum as well, though very rarely.

Informed Consent: Written consent was obtained from the parents of the patient.

Peer-review: Externally peer-reviewed.

Author Contributions: Concept - HY, GiB; Design - HY, GiB; Supervision - HY, GIB; Collection and/or Processing - HY, GiB; Analysis and/or Interpretation - HY, GiB; Literature Review - HY, GiB; Writing HY, GiB; Critical Review - HY, GiB.

Conflict of Interest: No conflict of interest was declared by the authors.

Financial Disclosure: The authors declared that this study has received no financial support. 


\section{References}

1. Alfajaro MM, Cho KO. Evidences and consequences of extra-intestinal spread of rotaviruses in humans and animals. VirusDisease 2014;25:18694.

2. Özalp E, Aydın Teke T, Tanır G, Özkan M, Bayhan Gi. Transient acute flaccid paralysis and seizures associated with rotavirus gastroenteritis in a child. Turk J Pediatr 2012;54:661-3.

3. Hemming $M$, Huhti L, Räsänen $S$, Salminen $M$, Vesikari T. Rotavirus antigenemia in children is associated with more severe clinical manifestations of acute gastroenteritis. Pediatr Infect Dis J 2014;33:366-71.

4. Litwin L, Machura E. The etiology and clinical manifestation of erythema nodosum in hospitalized children-analysis of 12 cases. Preliminary report. Dev Period Med 2014;18:506-12.

5. Wyllie R, Hyams JS, Kay M. Pediatric gastrointestinal and liver disease. In: Galloway DP, Cohen MB (eds). Infectious Diarrhea. Fifth ed. Chapter 38, 2016:441-62.
6. Esona MD, Gautam R. Rotavirus. Clin Lab Med 2015;35:363-91.

7. Schwartz RA, Nervi SJ. Erythema nodosum: a sign of systemic disease. Am Fam Physician 2007;75:695-700.

8. Acosta KA, Haver MC, Kelly B. Etiology and therapeutic management of erythema nodosum during pregnancy: an update. Am J Clin Dermatol 2013;14:215-22.

9. Aydın-Teke T, Tanır G, Bayhan Gl, Metin O, Oz N. Erythema nodosum in children: evaluation of 39 patients. Turk J Pediatr 2014;56:144-9.

10. Akelma AZ, Cizmeci MN, Mete E, Malli DD, Erpolat S, Sonmez FM. Macular exanthema in a child with rotavirus gastroenteritis: a case report. Arch Argent Pediatr 2014;112:e53-e56.

11. Di Lernia V, Ricci C. Skin manifestations with rotavirus infections. Int J Dermatol 2006;45:759-61.

12. Ozbagcivan $O$, Akarsu S, Avci C, Inci BB, Fetil E. Examination of the microbial spectrum in the etiology of erythema nodosum: a retrospective descriptive study. J Immunol Res 2017;2017:1-8. 